\title{
Characteristic Impedance of a Microstrip-Like Interconnect Line in Presence of Ground Plane Aperture
}

\author{
Rohit Sharma, T. Chakravarty, Sunil Bhooshan, and A. B. Bhattacharyya \\ Received 9 May 2007; Revised 27 August 2007; Accepted 17 December 2007 \\ Recommended by Chang-Ho Lee \\ We propose new empirical expressions for the characteristic impedance of a microstrip-like interconnect line in presence of ground \\ plane aperture. The existing characteristic impedance expressions are modified so as to include the effect of the ground plane \\ aperture. The variation in the characteristic impedance vis-à-vis the aperture size is established. The proposed expressions are \\ general and valid for a range of dielectric materials concerning MICs, RFICs, and PCBs. The results are validated by measurements \\ performed on a vector network analyzer.
}

Copyright (c) 2007 Rohit Sharma et al. This is an open access article distributed under the Creative Commons Attribution License, which permits unrestricted use, distribution, and reproduction in any medium, provided the original work is properly cited.

\section{INTRODUCTION}

Planar transmission lines form the basic media for integrated circuits and RF and PCB interconnections. These circuits can be fabricated in hybrid or monolithic form. The microstrip line is an inhomogeneous transmission line structure. It consists of a strip conductor on a flat dielectric substrate with a metallic ground plane on its reverse side. The characteristic impedance $Z$ of the microstrip line is given by $Z=Z_{a} / \sqrt{\varepsilon_{\text {eff }}}$, where $Z_{a}$ is the characteristic impedance of the microstrip line with the dielectric replaced by air [1-6]. Here, $\varepsilon_{\text {eff }}$ is the effective dielectric constant of the medium, and its value lies in the range $1<\varepsilon_{\text {eff }}<\varepsilon_{r}$, where $\varepsilon_{r}$ is the dielectric constant of the substrate. At low frequency, the mode of propagation resembles the TEM mode, and hence is termed as the quasi-TEM mode. Due to this quasi-TEM mode approximation, the calculation of propagation parameters of the line reduces to the solution of the two-dimensional Laplace's equation. A composite discussion is presented by the authors of [6]. Some of the reported methods for the solution of Laplace's equation include the conformal transformation method [7, 8], the variational method [7], and the finite difference method $[9,10]$. A simplified design of microstrip line is presented by Edwards [5], in closed-form expressions.

Traditionally, it is found that the characteristic impedance of a microstrip line is related to the width of the strip, the height of the substrate, and the dielectric constant of the substrate. However, with the introduction of a ground plane aperture (GPA) below the strip, the line properties can change significantly, in that the characteristic impedance varies with the width of the GPA. This aperture is formed by removing the ground plane below the microstrip line over the entire length of the line. The use of GPA has interesting applications like $3 \mathrm{~dB}$ edge coupler [11] and band pass filters [12]. A similar approach has been earlier presented [13], in that, an elevated coplanar waveguide structure has been presented and analyzed using FDTD simulation. Unlike [13], the present structure is not elevated coplanar waveguide, as in the present work, a new design equation is presented, which is valid for a wide range of substrates commonly used in MICs, PCBs, and RFICs.

In this paper, a new set of empirical expressions for computing the characteristic impedance of the microstrip-like interconnect line with GPA is proposed. Existing characteristic impedance formulae are modified to incorporate the effect of the aperture. The expressions are obtained using conventional numerical techniques applied to FDTD simulation results. The FDTD simulation is carried out using accurate and commercially available software, CST Microwave Studio. These expressions are general, in that they cover all dielectric materials commonly used for MICs, RFICs, and PCBs. The expressions are obtained using quasistatic approximation and are thus valid for $h / \lambda g<0.02$. The results obtained using these expressions are compared with FDTD simulations to check their accuracy and are validated by measurements performed on a vector network analyzer. The proposed formulation can be useful in the design and analysis of high-speed multichip module interconnects, as well as multilayer PCBs and RFICs with optimum signal integrity. Applications can also be found in millimeter-wave components 


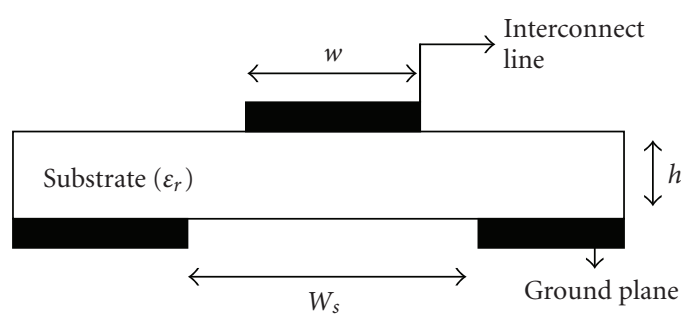

Figure 1: Microstrip-like interconnect line with ground plane aperture.

which require shorter electrical lengths, directional couplers, bandpass filters, and split rings.

The paper is organized as follows. In Section 2, we present the new set of expressions for characteristic impedance of a given microstrip interconnect line with GPA. The results obtained in this section are compared with FDTD simulations and measurements in Section 3. The paper concludes in Section 4.

\section{FORMULATION OF THE CHARACTERISTIC IMPEDANCE (Z)}

A standard microstrip line consists of a thin, flat conductor of width $(W)$ separated from a ground plane by a dielectric material of height $(h)$ and permittivity $\left(\varepsilon_{r}\right)$. The width of the strip $(W)$, the height of the substrate $(h)$, and the dielectric constant of the substrate $\left(\varepsilon_{r}\right)$ characterize the impedance $(Z)$ of this line and the effective dielectric constant $\left(\varepsilon_{\text {eff }}\right)$ of the substrate [1], and they are given by

$$
Z=\left\{\begin{array}{c}
\frac{60}{\sqrt{\varepsilon_{\mathrm{eff}}}} \ln \left(8 \frac{h}{W}+0.25 \frac{W}{h}\right) \mathrm{ohms} \\
\text { for } \frac{W}{h}<1, \\
\frac{120 \pi}{\sqrt{\varepsilon_{\mathrm{eff}}} \times[W / h+1.393+(2 / 3) \ln (W / h+1.44)]} \text { ohms } \\
\text { for } \frac{W}{h} \geq 1,
\end{array}\right.
$$

$$
\varepsilon_{\mathrm{eff}}=\frac{\varepsilon_{r}+1}{2}+\frac{\varepsilon_{r}-1}{2}\left(1+\frac{10 h}{W}\right)^{-1 / 2} .
$$

We now introduce an aperture in the ground plane below the line. Figure 1 shows the resultant interconnect structure used in this analysis. Throughout this study, the length of the GPA is considered to be constant and it is equal to the line length, with the width of the aperture, $W_{s}$, varied from zero to a finite value. It is observed that the characteristic impedance varies significantly as the GPA width is increased. Figure 2 shows the variation in the characteristic impedance with respect to the aperture width $\left(W_{s}\right)$. These results are obtained through FDTD simulations. The characteristic impedance $(Z)$ exhibits some sort of linear relationship with the aperture width $\left(W_{s}\right)$. Note that at $W_{s}=0$, the values reflect the impedance of a microstrip line. While performing the FDTD simulations, care has been taken to ensure that the frequency

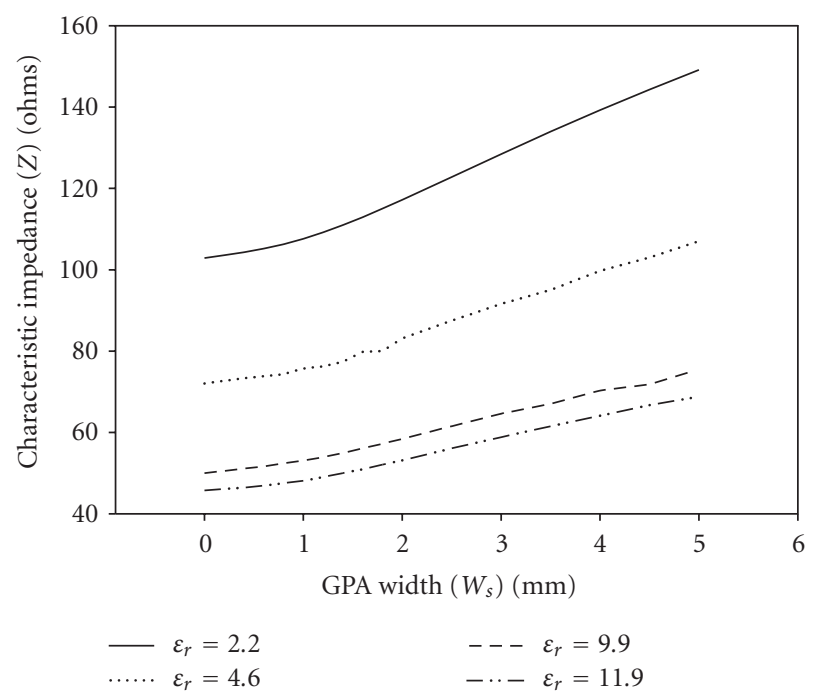

(a)

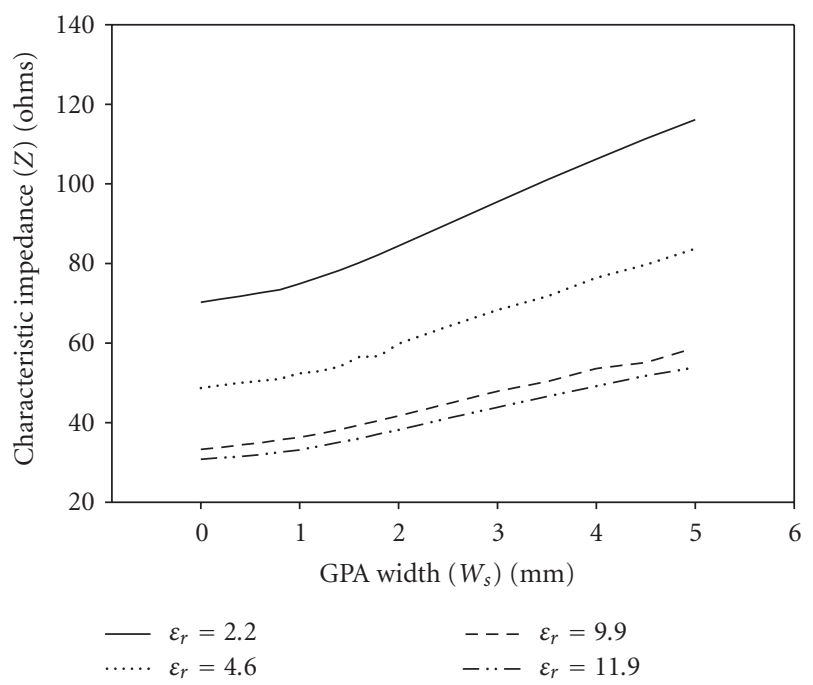

(b)

FIgURE 2: Characteristic impedance $(Z)$ as a function of GPA width $\left(W_{s}\right)$ obtained using FDTD simulation: (a) $W=0.42 \mathrm{~mm}, h=$ $0.508 \mathrm{~mm}, \varepsilon_{r}=2.2,4.6,9.9$, and 11.9; (b) $W=0.9 \mathrm{~mm}, h=$ $0.508 \mathrm{~mm}, \varepsilon_{r}=2.2,4.6,9.9$, and 11.9.

sweep is more than ten times the frequency of measurement. It is to be understood that quasistatic approximation is valid for electrically thin lines and the results plotted here are at frequencies less than $3 \mathrm{GHz}$.

Essentially, with the introduction of the GPA, the capacitance of the line decreases. This is shown by the field distribution in Figure 3. Therefore, there is substantial increase in characteristic impedance, which is given by $Z=1 / c \sqrt{C C^{a}}$ [6]. Here, $C$ is the capacitance per unit length of the structure, $C^{a}$ is the capacitance per unit length of the structure with all dielectrics replaced by air, and $c$ is the velocity of propagation in air. Based on this reasoning, the existing expressions given in (1) can be modified to incorporate this effect of the GPA. We introduce a new multiplying factor " $K$," 


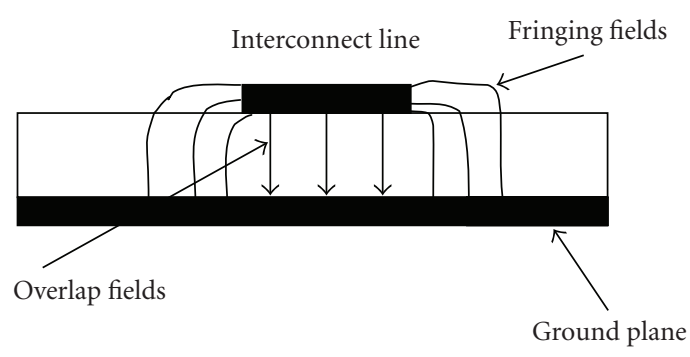

(a)

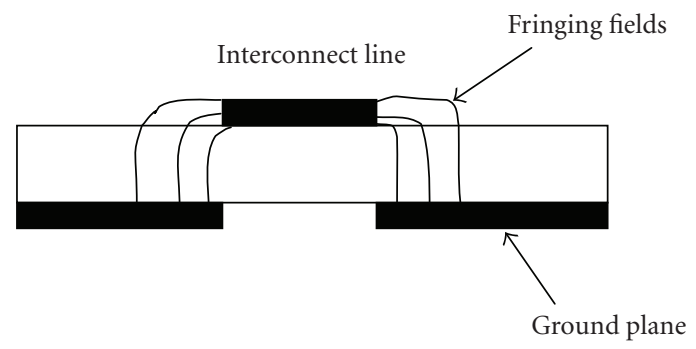

(b)

FIGURE 3: Field distribution in the interconnect line: (a) without ground plane aperture, and (b) with ground plane aperture.

which takes into consideration the effect of $W, h, W_{s}$, and $\varepsilon_{r}$ on line impedance. Using conventional numerical techniques, (1) is modified and is given by

$$
Z=\frac{60}{\sqrt{\varepsilon_{\mathrm{eff}}}} \ln \left(8 \frac{h}{W}+0.25 \frac{W}{h}\right) \cdot K \text { for } \frac{W}{h}<1,
$$

where

$$
\begin{aligned}
& K=0.99+ 0.22 W_{s}+0.00219 \varepsilon_{r}-0.0175 h-0.0994 W \\
&+0.000573 W_{s} \varepsilon_{r}+0.0716 W_{s} W-0.259 W_{s} h \\
&-0.00527 \varepsilon_{r} W-0.00359 \varepsilon_{r} h+0.111 W h \\
&+0.00991 W_{s} \varepsilon_{r} W-0.00058 W_{s} \varepsilon_{r} h \\
&+0.0073 \varepsilon_{r} W h-0.0113 W_{s} \varepsilon_{r} W h, \\
& Z=\frac{120 \pi \quad \text { for } \frac{W}{h} \geq 1,}{\sqrt{\varepsilon_{\mathrm{eff}}} \times[W / h+1.393+(2 / 3) \ln (W / h+1.44)]} \cdot K
\end{aligned}
$$

where

$$
\begin{aligned}
K=1.05 & +0.358 W_{s}-0.00184 \varepsilon_{r}-0.119 h-0.15 W \\
& +0.00505 W_{s} \varepsilon_{r}+0.0566 W_{s} W-0.474 W_{s} h \\
& +0.00571 \varepsilon_{r} W+0.00297 \varepsilon_{r} h+0.195 W h \\
& -0.00205 W_{s} \varepsilon_{r} W-0.00677 W_{s} \varepsilon_{r} h \\
& -0.00865 \varepsilon_{r} W h+0.00408 W_{s} \varepsilon_{r} W h .
\end{aligned}
$$

It should be noted that when the aperture width $\left(W_{s}\right)$ reduces to zero, the multiplying factor equates to unity and thus represents the original microstrip line impedance. In the

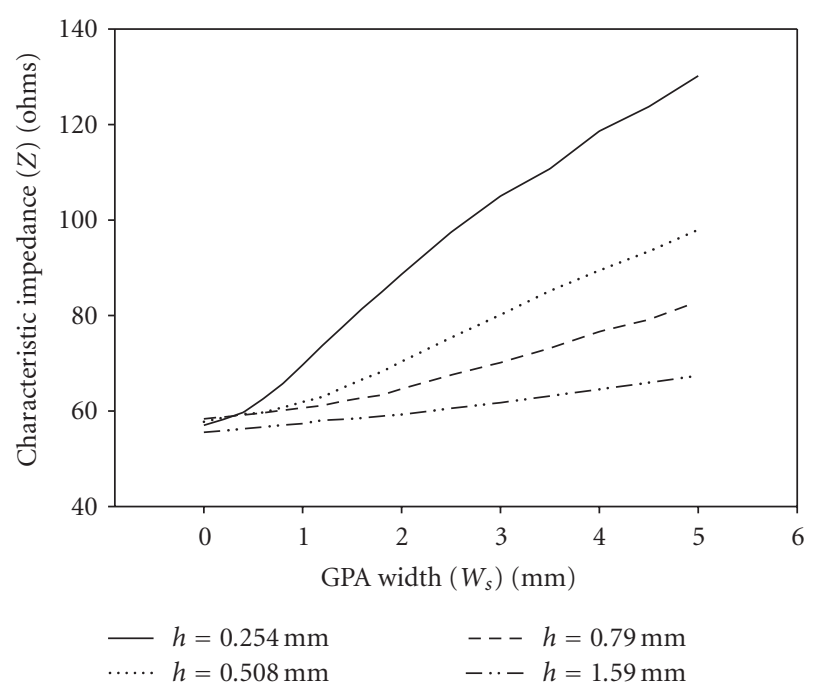

Figure 4: Characteristic impedance $(Z)$ for different substrate heights $(h): W / h=1.75, \varepsilon_{r}=3.2$, and $h=0.254,0.508,0.79$, and $1.59 \mathrm{~mm}$.

above expressions, we have studied the effect of $W / h, W_{s}, \varepsilon_{r}$ and all possible combinations of these parameters on the characteristic impedance $(Z)$, using stepwise multiple regression technique. The results obtained are valid for a wide range of materials $\left(2 \leq \varepsilon_{r} \leq 12\right)$ and $0<W / h \leq 3$. Using (3) and (5), one can obtain line impedance for a variety of substrate heights $(0.254 \leq h \leq 1.59 \mathrm{~mm})$. It may be of interest to the reader that for larger substrate heights, the change in the characteristic impedance $(Z)$ with respect to the aperture width $\left(W_{s}\right)$ is marginal. This is given in Figure 4 . Thus, the substrate heights $(h)$ listed above are sufficient to study the effect of aperture width $\left(W_{s}\right)$ on line impedance.

The multiplying factor " $K$ " obtained above is expressed directly in terms of the dielectric constant $\left(\varepsilon_{r}\right)$ of the substrate. The effective dielectric constant is given by $\varepsilon_{\text {eff }}=C / C^{a}$ [6]. With varying GPA width $\left(W_{s}\right)$, this ratio is unchanged as the values of $C$ and $C^{a}$ decrease by an almost similar amount. Thus, we may use the same expressions for $\varepsilon_{\text {eff }}$ as given in (2). Unlike the expressions proposed by Wheeler [2], the computation of $\varepsilon_{\text {eff }}$ is unimportant in our case. The characteristic impedance $(Z)$ can directly be calculated from the interconnect geometry (i.e., $W / h, W_{s}$, and $\varepsilon_{r}$ ).

\section{RESULTS}

We now compare results obtained from (3) and (5) with FDTD simulations as shown in Figure 5 . The results obtained above exhibit good accuracy and are valid for $h / \lambda_{g}<0.02$. The results are validated by measurements performed on a vector network analyzer. Figure 6 shows the fabricated structure. The fabrication is done on an FR4 substrate for three different aperture widths $\left(W_{s}\right)$.

It is seen that the prediction of characteristic impedance value for a line of given dimensions with GPA is accurate with $\pm 2 \%$ error as shown in Table 1 . 
TABLE 1: Characteristic impedance $Z$ obtained by measurements and proposed formulation $\left(\varepsilon_{r}=4.6, W=4 \mathrm{~mm}, h=0.79 \mathrm{~mm}\right)$.

\begin{tabular}{lccc}
\hline GPA width $\left(W_{s}\right)$ & Measured results & FDTD simulation results & Proposed results \\
\hline $3 \mathrm{~mm}$ & $38.58 \Omega$ & $37.87 \Omega$ & $38.18 \Omega$ \\
$4 \mathrm{~mm}$ & $43.97 \Omega$ & $43.16 \Omega$ & $43.52 \Omega$ \\
$5 \mathrm{~mm}$ & $49.21 \Omega$ & $47.68 \Omega$ & $48.86 \Omega$ \\
\hline
\end{tabular}

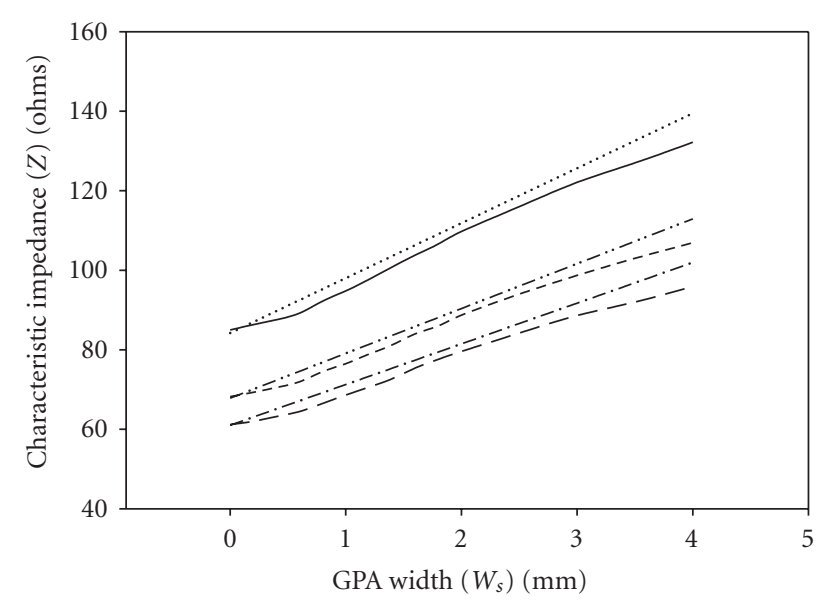

- Simulated $\left(\varepsilon_{r}=6\right)$

…... Proposed $\left(\varepsilon_{r}=6\right)$

--- Simulated $\left(\varepsilon_{r}=9.5\right)$

$-\cdot-$ Proposed $\left(\varepsilon_{r}=9.5\right)$

- - Simulated $\left(\varepsilon_{r}=11.9\right)$

-. . Proposed $\left(\varepsilon_{r}=11.9\right)$

(a)

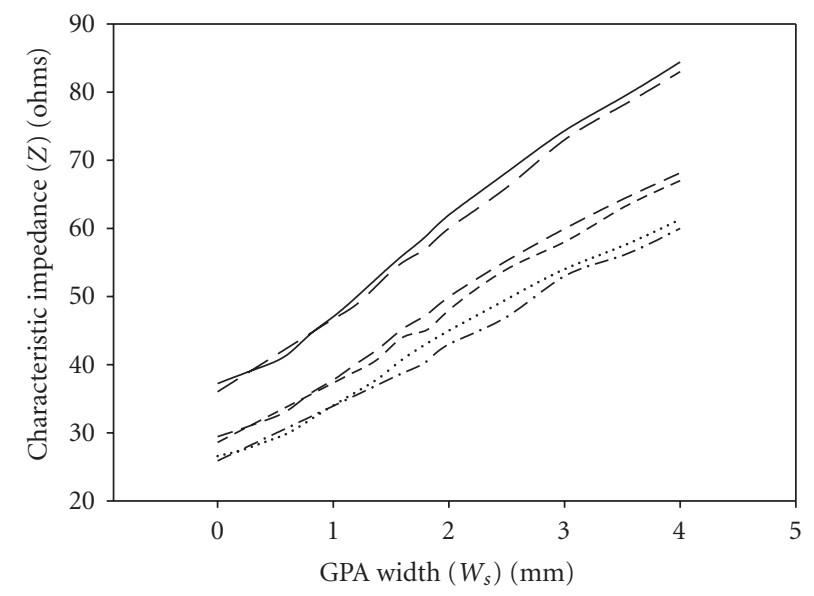

$$
\begin{array}{ll}
\text { - Simulated }\left(\varepsilon_{r}=6\right) & --. \text { Proposed }\left(\varepsilon_{r}=9.5\right) \\
-- \text { Proposed }\left(\varepsilon_{r}=6\right) & \text {...... Simulated }\left(\varepsilon_{r}=11.9\right) \\
-- \text { Simulated }\left(\varepsilon_{r}=9.5\right) & \text {-.-. Proposed }\left(\varepsilon_{r}=11.9\right)
\end{array}
$$

(b)

FIgURE 5: Characteristic impedance $(Z)$ obtained using proposed formulation and FDTD simulations: (a) $W / h=0.5$ and $\varepsilon_{r}=6,9.5$ and 11.9; (b) $W / h=2.25$ and $\varepsilon_{r}=6,9.5$ and 11.9 .

\section{CONCLUSION}

The analysis of the proposed structure can also be carried out using conformal mapping or variational analysis techniques. However, these methods are computationally rigorous. Just like Wheeler's presentation, in this paper, an attempt has

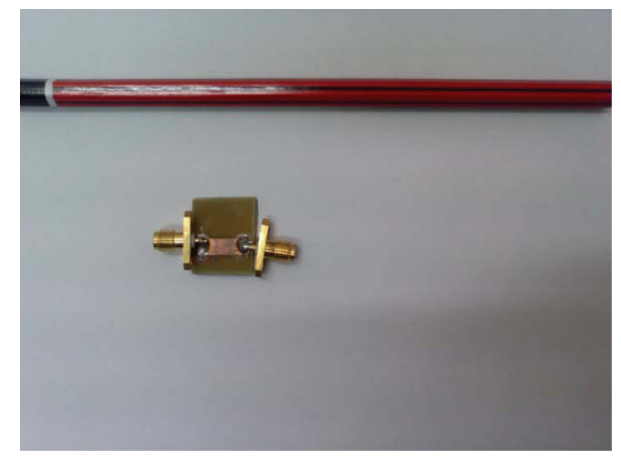

(a)

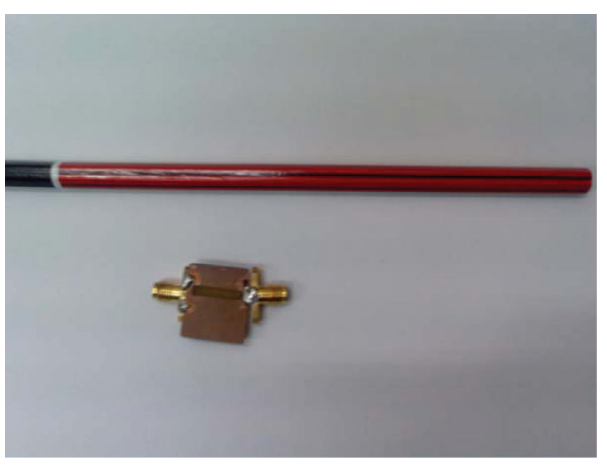

(b)

FIGURE 6: Fabricated interconnect structure: (a) interconnect line and (b) ground plane aperture: $W=4 \mathrm{~mm}, h=0.79 \mathrm{~mm}, \varepsilon_{r}=$ 4.6, and $W_{s}=3 \mathrm{~mm}$.

been made to give closed-form expressions for evaluating characteristic impedance of the line with GPA. It is envisaged that these equations will find their utility as a first-order, reasonably accurate synthesis step. The comparison between the simulated results and the proposed formulation shows good agreement. The proposed formulations will form a very useful tool in designing MCM and RF interconnections with excellent accuracy. This method is useful in the fabrication of high impedance lines.

\section{REFERENCES}

[1] H. A. Wheeler, "Transmission-line properties of parallel strips separated by a dielectric sheet," IEEE Transactions on Microwave Theory and Techniques, vol. 13, no. 2, pp. 172-185, 1965.

[2] H. A. Wheeler, "Transmission-line properties of parallel wide strips by a conformal-mapping approximation," IEEE 
Transactions on Microwave Theory and Techniques, vol. 12, no. 3, pp. 280-289, 1964.

[3] H. A. Wheeler, "Transmission-line properties of a strip on a dielectric sheet on a plane," IEEE Transactions on Microwave Theory and Techniques, vol. 25, no. 8, pp. 631-647, 1977.

[4] E. Yamashita and R. Mitra, "Variational method for the analysis of microstrip lines," IEEE Transactions on Microwave Theory and Techniques, vol. 16, no. 4, pp. 251-256, 1968.

[5] T. C. Edwards, Foundations of Microstrip Circuit Design, John Wiley \& Sons, New York, NY, USA, 1981.

[6] B. Bhat and S. K. Koul, "Unified approach to solve a class of strip and microstrip-like transmission lines," IEEE Transactions on Microwave Theory and Techniques, vol. 82, no. 5, pp. 679-686, 1982.

[7] L. A. Pipes, Applied Mathematics for Engineers and Physicists, McGraw-Hill, New York, NY, USA, 1958.

[8] R. E. Collin, Field Theory of Guided Waves, McGraw-Hill, New York, NY, USA, 1960.

[9] H. E. Green, "The numerical solution of some important transmission-line problems," IEEE Transactions on Microwave Theory and Techniques, vol. 13, no. 5, pp. 676-692, 1965.

[10] M. V. Schneider, "Computation of impedance and attenuation of TEM-lines by finite difference methods," IEEE Transactions on Microwave Theory and Techniques, vol. 13, no. 6, pp. 793800, 1965.

[11] R. Sharma, T. Chakravarty, S. Bhooshan, and A. B. Bhattacharyya, "Design of a novel $3 \mathrm{db}$ microstrip backward wave coupler using defected ground structure," Progress in Electromagnetics Research, vol. 65, pp. 261-273, 2006.

[12] M. K. Mandal and S. Sanyal, "Design of wide-band, sharprejection bandpass filters with parallel-coupled lines," IEEE Microwave and Wireless Components Letters, vol. 16, no. 11, pp. 597-599, 2006.

[13] S. Hofschen and I. Wolff, "Simulation of an elevated coplanar waveguide using 2-D FDTD," IEEE Microwave and Guided Wave Letters, vol. 6, no. 1, pp. 28-30, 1996.

\section{AUTHOR CONTACT INFORMATION}

Rohit Sharma: Jaypee University of Information Technology, Waknaghat, Solan 173215, India; rohit.sharma@juit.ac.in

T. Chakravarty: Embedded Systems Innovation Lab, Tata Consultancy Services, Bangalore 560066, India; tapas.chakravarty@tcs.com

Sunil Bhooshan: Jaypee University of Information Technology, Waknaghat, Solan 173215, India; sunil.bhooshan@juit.ac.in

A. B. Bhattacharyya: Jaypee Institute of Information Technology, Noida 201307, India; ab.bhattacharya@jiit.ac.in 

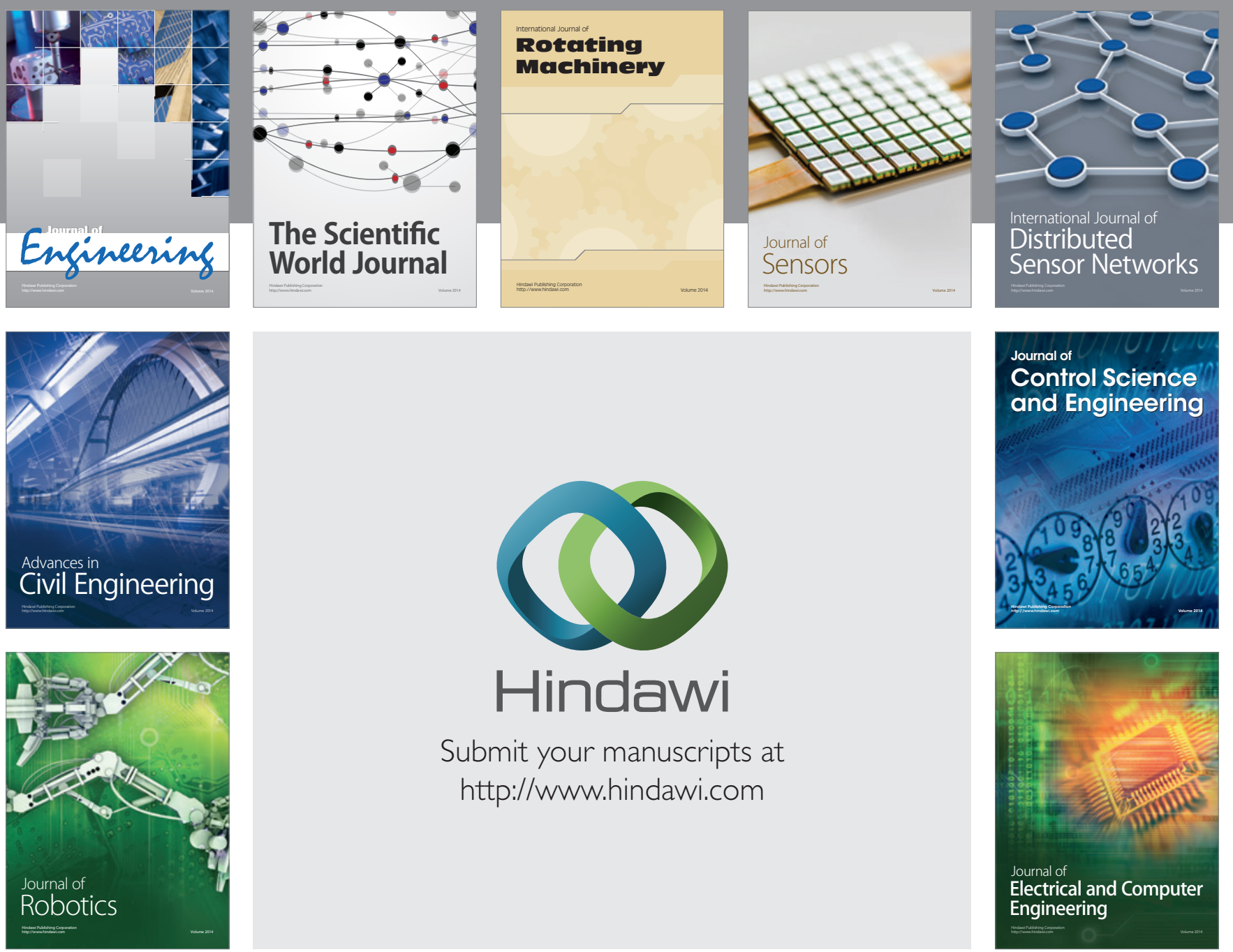

Submit your manuscripts at

http://www.hindawi.com
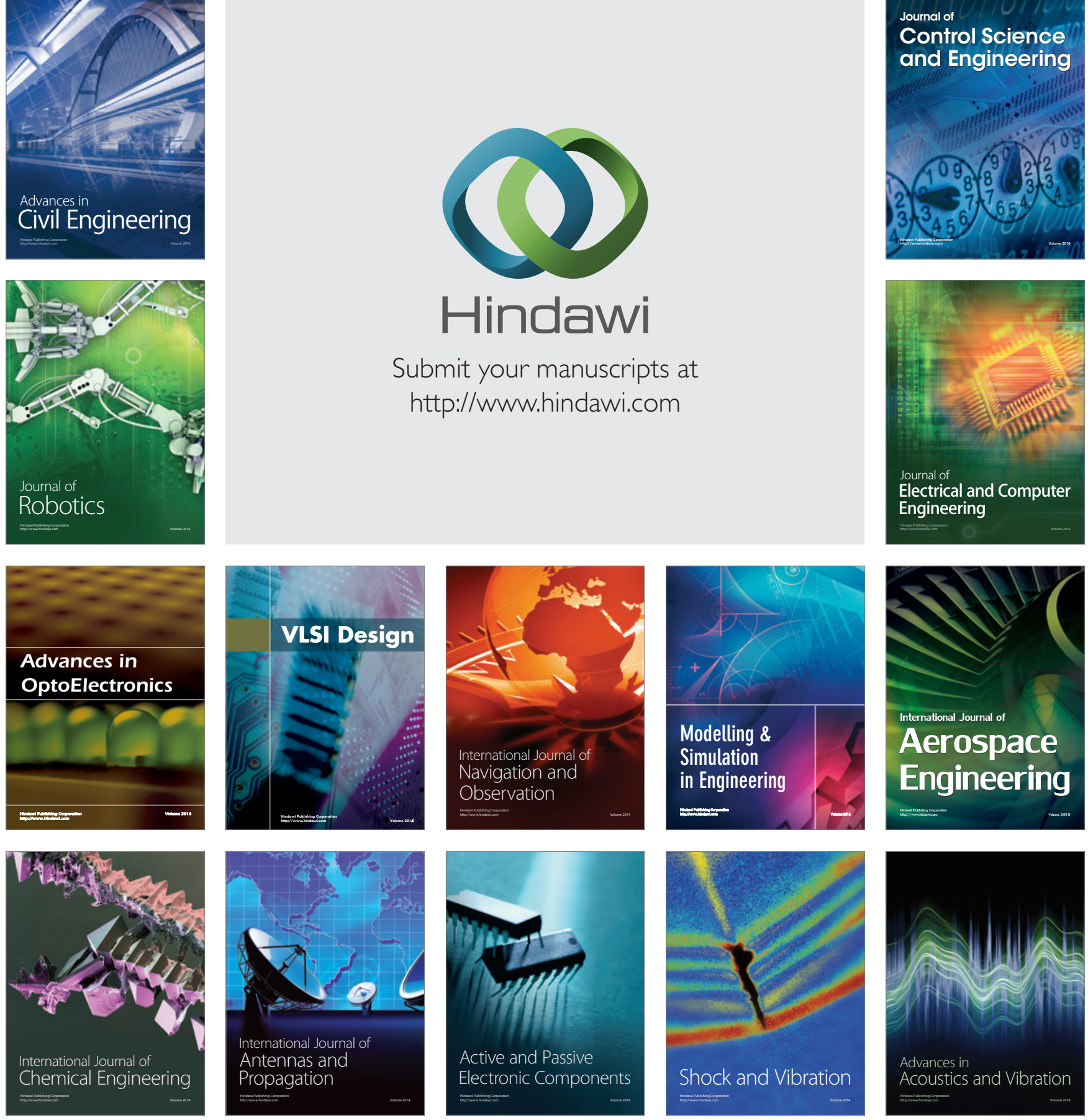